\title{
Impact of Synchrophasor Estimation Algorithms in ROCOF-based Under-Frequency Load-Shedding
}

\author{
Yihui Zuo, Student Member, IEEE, Guglielmo Frigo, Member, IEEE, Asja Derviškadić, Member, IEEE, \\ Mario Paolone, Senior Member, IEEE
}

\begin{abstract}
This paper investigates the impact of synchrophasor estimation algorithms in Under-Frequency Load-Shedding (UFLS) and Load-Restoration (LR) schemes, relying on frequency and Rate-of-Change-of-Frequency (ROCOF) measurements produced by Phasor Measurement Units (PMUs). We compare two consolidated window-based synchrophasor estimation algorithms, as representative approaches based on static and dynamic signal models, with a focus on the appropriateness of utilizing PMU-based ROCOF measurements. The performance of the proposed relaying scheme is assessed by means of a Real-Time Simulator implementing the time-domain full-replica dynamic model of the IEEE 39-Bus power system.
\end{abstract}

Index Terms-Under-Frequency Load-Shedding (UFLS); Phasor Measurement Units (PMUs); IEEE 39-Bus; Real-Time Digital Simulation; Rate-of-Change-of-Frequency (ROCOF).

\section{INTRODUCTION}

As known, Under-Frequency Load-Shedding (UFLS) is a technique that minimizes the risk of uncontrolled system separation, loss of generation, or shutdown in case of large power system disturbances, after the primary frequency control reserve is exhausted [1]. Typically, UFLS schemes rely on frequency estimates, as computed over extended time-intervals $(\sim 1$ s) in order to maximize the estimation accuracy [2]. During a large contingency, if the frequency exceeds a specific threshold, dedicated relays automatically trip selected loads in order to preserve grid interconnections and generation capability [1]-[3]. If a sufficient amount of loads are shed, then the system operation can be smoothly restored.

Modern power systems are characterized by large shares of inverter-connected resources, that do not provide any inertia and thus lead to extremely fast dynamics in case of sudden contingencies. A clear example happened in September 2016 when the South Australian system faced a severe blackout when a wind storm hit the region while half of the power consumption was fed by wind generation [4]. The frequency experienced a large drop of almost $4 \mathrm{~Hz}$ in less than 1 second, with an estimated Rate-of-Change-of-Frequency (ROCOF) of roughly $6.25 \mathrm{~Hz} / \mathrm{s}$. In this context, the extended time-intervals employed by typical UFLS schemes are incompatible with such dynamics.

The authors are with the École Polytechnique Fédérale de Lausanne EPFL, CH-1015, Lausanne, Switzerland.

This work was supported in part by the Swiss Centre for Competence in Energy Research on the Future Swiss Electrical Infrastructure (SCCER) through Swiss Innovation Agency (InnosuisseSCCER Program) and part by European Union's Horizon 2020 Research and Innovation Program under Grant 773406 .
For this reason, the employment of Phasor Measurement Units (PMUs) might represent a promising solution thanks to their remarkable measurement accuracy as well as time synchronization [5], [6]. Indeed, PMUs are able to provide frequency and ROCOF estimates with a reporting rate in the order of tens of frames per second [7], [8]. Since they rely on a static signal model, PMU-based measurements may underperform in time-varying conditions, particularly if transient events occur [9], [10]. As a consequence, their measurement uncertainty has to be taken into account within the control scheme [11], [12]. In [12], the authors investigate the feasibility of PMU deployment in ROCOF-based applications, focusing on the problem of ROCOF measurement in terms of estimation accuracy and reliability. Nevertheless, since ROCOF is defined as the frequency first-order time-derivative, it can be seen as a predictive filter whose output accounts for the variation polarity and velocity.

Traditionally, the approaches for determining the amount of shed loads are based on the sole use of frequency [1], [2]. Indeed, after a certain disturbance took place, the generationload mismatch can be estimated by analyzing the frequency response characteristic [13]. By monitoring the frequency disturbances in several grid nodes (e.g., via PMUs), it is possible to detect ongoing transients and determine the most suitable counteraction [14], [15]. In this regard, it is worth noticing that the connection, or disconnection, of a load produces rapid transients that affect the PMUs' estimation accuracy [16]. In addition, when dealing with large power networks, it might be beneficial to partition the analysis in several grid areas in order to reduce the computational complexity. In this case, though, the relationship between different control actions in different grid areas has to be considered [17].

More sophisticated approaches still rely on frequency measurements, but they determine the amount of load to be shed based on an estimation of the active power deficiency. These methods are also called adaptive, because the location, speed and amount of shed loads are adjusted adaptively based on the specific contingency evolution [3]. To the best of Authors' knowledge, though, there exist only few UFLS schemes that adopt ROCOF thresholds to trigger the LS action [18]-[20]. Mostly, they analyze and acknowledge the necessity of using ROCOF as an index for UFLS schemes, without providing a strategy to actually measure it. Indeed, frequency and ROCOF are computed using a simplified system frequency response model or by assuming the information of the studied power grid is fully available, rather than using actual measuring devices (e.g., synchrophasors). 
In this context, the disturbance magnitude can be identified from ROCOF measurements, as they are assumed to be proportional to the power shortage size through the inertia constant: the higher the ROCOF, the more loads need to be shed [21]-[23]. An alternative approach employs the instantaneous voltage deviation as a criterion of proximity to the failure point: the buses nearby the failure place experience larger voltage reduction and should be discarded first [24]-[26]. To guarantee a smoother system restoration, these approaches introduce significant time delays between consecutive actions. The combined effect of measurement and delay time makes the control schemes more robust, but also less responsive and prompt to react to fast dynamics as observed in [4].

Within this context, this paper investigates the impact of synchrophasor estimation algorithms in ROCOF-based UFLS schemes. In more details, the contributions of this paper are listed here below.

- First, we analyze the anticipative property of ROCOF with respect to frequency in detecting large electromechanical transients. This may potentially lead to a faster restoration to a new equilibrium point, and, therefore, leveraged by the proposed relaying strategy.

- Then, we propose an effective local UFLS and LoadRestoration (LR) scheme, based on ROCOF and frequency measurements as provided by PMUs. We further present how to properly select the strategy's parameters based on the targeted power system. Two sets of ROCOF thresholds are studied, in order to assess the impact of the parameters tuning.

- We analyze the impact of the synchrophasor estimation algorithms on the proposed ROCOF-based UFLS, by comparing two consolidated window-based synchrophasor estimators. The first one is based on a static signal model [27], the second one is based on a dynamic signal model [28]. As their estimation accuracy has been thoroughly characterized in previous publications, in this paper we mainly focus on the impact of ROCOF computation on the UFLS performance.

- We assess the performance of the proposed relaying scheme by means of a OPAL-RT Real-Time Simulator (RTS), where we reproduce a full-replica of the timedomain dynamic model of the IEEE 39-Bus power system, hosting substantial amount of distributed energy resources [29], [30]. The model used for the study is available open-source.

The paper is structured as follows. Section II introduces the context of PMU-based ROCOF measurements. Section III provides details of the proposed UFLS and LR scheme. Section IV describes the power grid model used in the real-time simulation. Section V presents the performance validation, including simulation scenarios, results and discussion. Finally, Section VI summarizes the conclusions of the paper.

\section{PMU-BASED ROCOF MEASUREMENTS}

In general, the traditional control scheme of ROCOF-based UFLS relies on four main steps. First, the acquired waveform is processed in order to extract the fundamental frequency.
Based on this information, the ROCOF is computed as the first-order time-derivative. Then, a low-pass filtering stage removes fast and noisy ROCOF dynamics, thus providing a smoother trend, besides introducing an inevitable time delay. Finally, the obtained measurements are compared with given thresholds, whose excess activates the control actions.

In this context, the recent literature provides several solutions, but it is not possible to identify a list of common guidelines. The frequency estimation stage should consider a window length in the order of seconds, in view of higher resolution and noise rejection. The low-pass filtering stage is typically implemented as a moving average, whose window length has to be suitably scaled based on the expected ROCOF variability. However, such time delays are not compatible with the typical dynamics of modern power systems, e.g. [4].

Furthermore, during large electromechanical transients, power exchanges are taking place in a broad spectrum, well beyond the single fundamental component, therefore, the definition of frequency and ROCOF associated to the fundamental component represents an open issue from the metrological point of view [9]-[12], [31]-[33].

In a PMU-based measurement scenario, instead, the IEEE Std C37.118.1 introduces stringent limits in terms of reporting latency that make it difficult to perform the ROCOF estimation over window lengths that account for three and five nominal cycles, i.e. 60 and $100 \mathrm{~ms}$ at $50 \mathrm{~Hz}$, for P- and M-class, respectively [7]. M-class is intended for measurement applications requiring accurate synchrophasor estimates, whereas $\mathrm{P}$-class is intended for mission-critical applications requiring fast responsiveness. As regards the estimation accuracy, RFE (Rate-of-Change-of-Frequency Error) is required not to exceed $0.01 \mathrm{~Hz} / \mathrm{s}$ in steady-state conditions, and $6 \mathrm{~Hz} / \mathrm{s}$ in the presence of harmonic distortion [8]. However, ROCOF measurements applied for real-world scenarios require a metering infrastructure more resilient against interfering components and characterized by a wider dynamic range. With respect to the South Australian blackout, the level of accuracy imposed by the IEEE Std C37.118.1 has the same order of magnitude of the values that are being measured. Also the reporting latency should not exceed few tens of ms, since the frequency drop is extremely fast. In other words, PMUs should be able to provide fast and accurate ROCOF estimates independently from the variation speed of the fundamental frequency or the distortion level [34].

It is worth to point out that, typically, PMUs rely on the time reference made available by the Global Positioning System (GPS) that represents an optimal trade-off between performance and installation cost [35]. However, in case the time reference is lost, PMUs might provide an erroneous synchrophasor estimation. Given the potential vulnerability of GPS to spoofing and jamming, PMUs adopted for missioncritical applications should use multiple timing sources, for instance deployable over the legacy power system telecommunication infrastructure [36]. Also, time drift and angle compensation strategies could be integrated into PMU devices, in order to reduce measurement errors deriving from potential timing source loss [37]. Nevertheless, it is worth noting that a GPS-disciplined oscillator, when free-running, presents a quite 
constant Allan deviation up to 10 minutes. That is to say that even if the GPS signal is lost, the free-running oscillator performance keeps sufficiently good for an amount of time much larger than the one typically required to reconnect to GPS. For this reason, this issue is considered negligible in an UFLS scenario like the one considered in the current paper.

Compared to traditional ROCOF relays, PMUs are specifically designed to produce frequently updated measurements with a reporting rate in the order of tens of fps. Typically, they consider short window lengths and they do not apply any moving average compensation. As a consequence, PMU estimates account for the quasi-instantaneous voltage and current variations, but their accuracy tends to deteriorate in the presence of dynamic trends or disturbances [10].

In this paper, we consider two PMU-based ROCOF estimation techniques, based on two consolidated state-of-theart algorithms, i.e. the Enhanced Interpolated DFT (e-IpDFT) [27] and the Compressive Sensing-based Taylor-Fourier Model (cs-TFM) [28]. We selected those two algorithms, because their implementation details are fully presented in the current literature, thus ensuring the replicability of the obtained results. The selected algorithms adopt not only a different processing approach, but also rely on a different signal model, i.e. static for e-IpDFT and dynamic for cs-TFM. The static estimation technique computes ROCOF as the incremental ratio between two consecutive frequency estimates. It is thus reasonable to expect that the ROCOF estimates are partially delayed and smoothed depending on the adopted reporting period. Conversely, the dynamic estimation technique is able to directly compute the instantaneous ROCOF as the secondorder time-derivative of phase. In general, the adoption of a dynamic signal model allows for tracking possible timevarying trends, but at the same time suffers from higher sensitivity to uncompensated disturbances [38].

\section{ROCOF-BASED LOAD SHEDDING}

a) On the Anticipative Effects of ROCOF: In this Section, we analyze the anticipating property of ROCOF measurements in detecting electro-mechanical transients with respect to frequency estimates. The study is meant to give a qualitative insight, because a quantitative and thorough study is griddependant and may only be provided via complex numerical simulations (see Section IV and V). For the sake of brevity, we here assume simplified models for rotating machines, loads, and network elements.

We consider a power grid in steady-state conditions, where $N$ synchronous machines have the same electrical angular speed $\Omega_{s}$. During electro-mechanincal transients, the rotating machine electro-mechanical power balance is expressed by the following well-know system of equations (e.g., [39]):

$$
\left\{\begin{array}{l}
\frac{d \Omega_{i}}{d t}=\frac{1}{M_{i}} \cdot\left(P_{m i}-P_{e i}\right) \\
\frac{d \delta_{i}}{d t}=\Omega_{i}-\Omega_{s}
\end{array}\right.
$$

where the index $i$ denotes the considered synchronous machine, whereas $\Omega_{i}$ and $\delta_{i}$ are its angular speed and angular position with respect to a reference machine rotating at $\Omega_{s}$. The terms $P_{m i}$ and $P_{e i}$ represent the mechanical driving power and the generated active electrical power, respectively, whereas $M_{i}$ defines the machine's inertia coefficient. This set of differential equations shows, as known, how the timederivative of the angular speed $d \Omega / d t$, i.e. the ROCOF, is proportional to the power imbalance in the grid.

Furthermore, as discussed in [40], in a power grid composed of $N$ generation buses and $M$ load buses, a change $\Delta P_{j}$ of active power at the $j$ th load bus causes a change $\Delta P_{e i}$ of active power at the $i$ th generation unit, given by:

$$
\begin{aligned}
\Delta P_{e i} & =\frac{C_{j i} \Delta P_{j}}{\sum_{i \in N} C_{j i}} \\
C_{j i} & =\frac{\left|\mathbf{K}_{i j} \mathbf{V}_{G}\right| \cos \theta_{j i}}{\left|\tilde{E}_{j}^{e q}\right|}
\end{aligned}
$$

where $\mathbf{K}$ is an $N \times M$ matrix obtained from system admittance matrix, $V_{G}$ is the generator bus voltage vector, $\tilde{E}_{j}^{e q}=\mathbf{K}_{j i} \mathbf{V}_{G}(j)$, and $\theta_{i j}$ is the angle between $\tilde{E}_{j}^{e q}$ and $\mathbf{K}_{j i} \mathbf{V}_{G}(i)$, and $\tilde{E}_{j}^{e q}$ denotes the Thevenin equivalent voltage at bus $j$.

The combination of (1), (2), and (3) provides an insight on why a ROCOF estimator might be used for a prompt and anticipative load shedding policy. Indeed, when acquiring ROCOF measurements from PMUs, that by definition are characterized by low reporting latency, we are able to infer any large power imbalance much faster than when using frequency. Thereby, we may immediately act on $\Delta P_{j}$ (i.e., load shedding) to counteract the power supply reduction (i.e., $P_{m i}-P_{e i}$ ).

b) The Proposed ROCOF-based UFLS Scheme: The proposed ROCOF-based UFLS scheme is inspired from [41] and consists of two parts: the ROCOF-based Load Shedding $(R$-LS) and the Frequency-based Load Restoration ( $f$-LR). This dual mechanism has been designed and optimized in order to ensure a fast reaction to power shortage as well as a secure network-restoration process. In particular, the $f$-LR thresholds have been derived from the guidelines in [1].

As shown in Table I, the control action is scaled to the threshold level, i.e. larger ROCOF and frequency values correspond to larger amounts of loads to be shed or restored, respectively. For this analysis, we consider two sets of ROCOF thresholds, briefly $R$-LS-1 and $R$-LS-2, and we compare the scheme performance as function of different load shedding shares.

As shown in Algorithm 1, in $R$-LS case, an activation criterion has to be satisfied. For each ROCOF threshold $R$, we evaluate the probability $p$ of exceeding $R$. If $p$ is larger than the activation level, the corresponding load share is activated. In the presence of multiple thresholds that are simultaneously activated, only the most severe control action is implemented, i.e. the largest share of loads is shed.

For the purpose of a smooth and stable system restoration, we introduce time-delays between two consecutive control actions. As regards $f$-LR, a suitably scheduled restoration avoids the occurrence of system instabilities or load shedding repetitions. To this end, in this paper we consider a time-delay of $5000 \mathrm{~ms}$, as recommended by [42].

However, to design a ROCOF-LS relay that uses ROCOF measurements from PMUs, we need to consider the limitation 


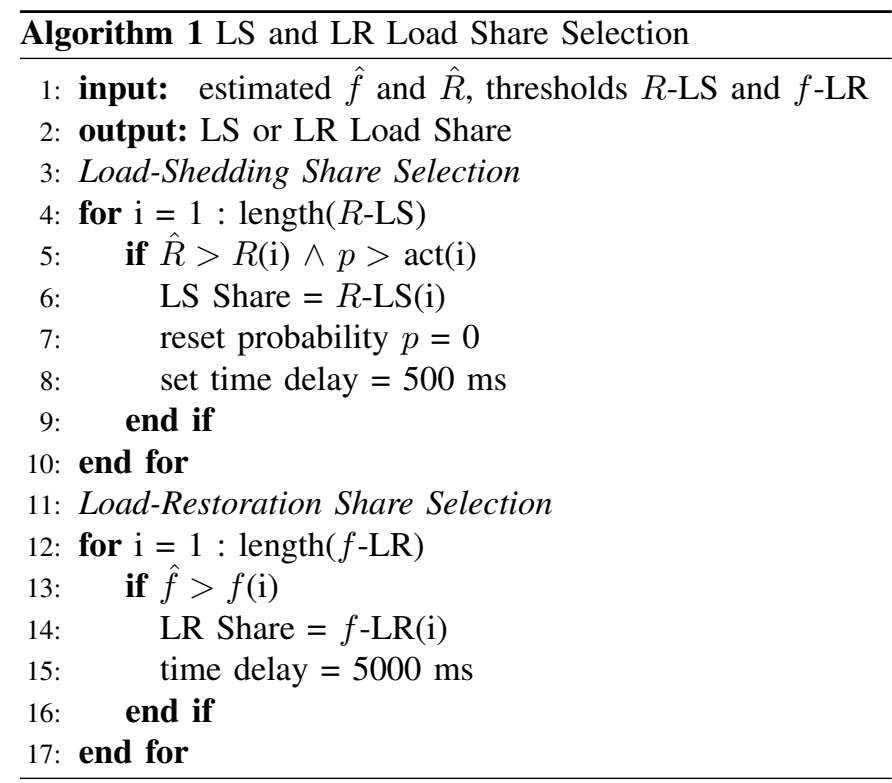

TABLE I

ROCOF AND FREQUENCY THRESHOLDS FOR LS AND LR

\begin{tabular}{cc|ccccccc}
\hline \multicolumn{2}{c}{ Load Share [\%] } & 100 & 95 & 90 & 85 & 75 & 60 & 50 \\
\hline \hline \multirow{2}{*}{ R-LS-1 } & $R$ [Hz/s] & & 0.3 & 0.4 & 0.6 & 0.7 & 1 & 1.2 \\
& $p_{\text {act }[\%]}$ & & 88 & 84 & 72 & 68 & 64 & 64 \\
\multirow{2}{*}{ R-LS-2 } & $R$ [Hz/s] & & 0.2 & 0.3 & 0.5 & 0.7 & 1 & 2 \\
& $p_{\text {act }}[\%]$ & & 84 & 60 & 60 & 60 & 52 & 52 \\
\hline \multirow{2}{*}{ f-LR } & $f[\mathrm{~Hz}]$ & 49.75 & 49.6 & 49.5 & 49.4 & 49.2 & 49 & \\
\hline
\end{tabular}

on the ROCOF estimation given by PMUs. Indeed, after the contingency, it is reasonable to expect that the voltage signal is affected by amplitude and phase modulations. The combined effect of amplitude and phase modulations is evident in both voltage waveform and corresponding ROCOF measurements. To this end, we consider ROCOF estimates $\hat{R}$ over an observation window interval of $500 \mathrm{~ms}$, as recommended in [42]. Given a PMU reporting rate of 50 frames per second, this corresponds to a set of 25 consecutive ROCOF estimates.

As shown in Table I, the ROCOF-LS relay embeds ROCOF thresholds and probability thresholds. For each ROCOF threshold $R$, the load shedding will be triggered if the probability $p$ of exceeding $R$ is larger than the activation threshold $p_{a c t}$. It is worth noticing that the load share is defined as a percentage of the installed load. It is also worth pointing out that the adopted ROCOF $R$ and activation thresholds $p_{a c t}$ are griddependant and can be obtained through dedicated sensitivity studies. In this respect, a strategy to tune these parameters, as well as a quantitative analysis on the interference of voltage modulation, are proposed in Section IV by making reference to the targeted electrical grid, i.e., the modified IEEE 39-bus.

c) UFLS Scheme Implementation: The ROCOF-based UFLS scheme is local, i.e., the relays located in different nodes do not exchange information and apply the control policy only based on the locally measured ROCOF and frequency value.

Within the simulated three-phase system, we assumed to install in each load bus a PMU that measures the bus voltage

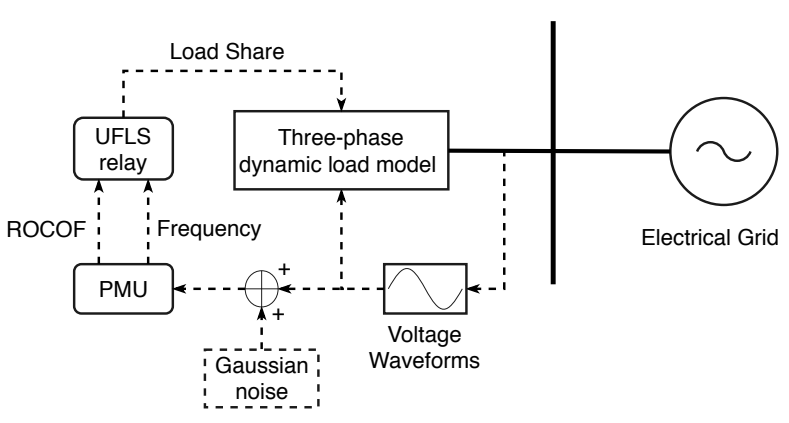

Fig. 1. Diagram of the UFLS scheme coupling with the dynamic load model.

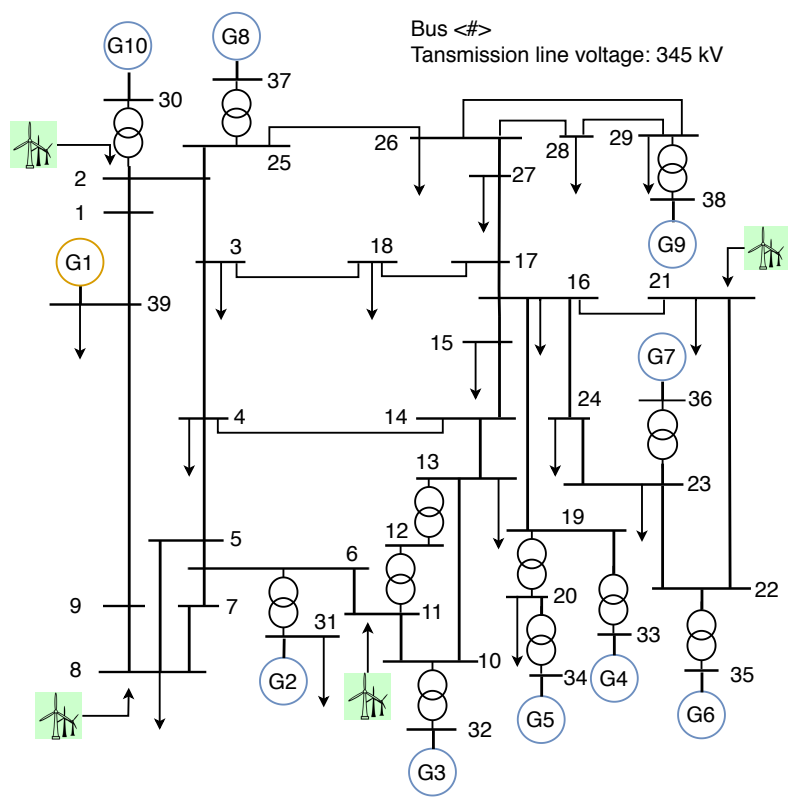

Fig. 2. Diagram of the modified IEEE 39-Bus power system.

amplitude, frequency and ROCOF associated to phase A. As shown in Fig. 1, the voltage waveform acquired by the PMU is corrupted by an additive white Gaussian noise, resulting in a Signal-to-Noise Ratio (SNR) of $80 \mathrm{~dB}$, in order to reproduce a plausible measurement noise. Once completed the estimation process, the PMU streams the measured ROCOF and frequency to the UFLS relay that determines the LS or LR load share based on the thresholds in Table I.

\section{Simulation MOdel}

The impact of the considered synchrophasor estimation algorithms on the performance of the proposed ROCOF-based UFLS plan is demonstrated through a full-replica dynamic model adapted from the IEEE 39-Bus standard test system [29], [43]. As shown in Fig. 2, the considered power grid consists of 39 buses with 19 loads, 10 synchronous generators, and 4 wind generators. In particular, the 4 wind farms (overall capacity of $1.35 \mathrm{GW}$ ) account for the increasing penetration of renewable generation in modern power grids.

For each synchrophasor estimation algorithm, we develop a dedicated PMU model to be deployed in every load bus [44], [45]. The considered power grid as well as the measurement devices are modelled in MATLAB Simulink and executed 
TABLE II

LIST OF GENERATION UNITS

\begin{tabular}{cccc}
\hline $\begin{array}{c}\text { Generation } \\
\text { Unit }\end{array}$ & $\begin{array}{c}\text { Plant } \\
\text { Type }\end{array}$ & $\begin{array}{c}\text { Installed } \\
\text { Capacity [MVA] }\end{array}$ & $\begin{array}{c}\text { FR } \\
\text { /Location }\end{array}$ \\
\hline \hline G1 & Thermal Plant & 3000 & PFR, SFR \\
G2-G4, G6-G10 & Hydro Plant & 1000 & PFR \\
G5 & Hydro Plant & 520 & PFR \\
\hline Wind Farm 1 & & 300 & bus 2 \\
Wind Farm 2 & Type-3 DFIG & 150 & bus 21 \\
Wind Farm 3 & & 400 & bus 8 \\
Wind Farm 4 & & 500 & bus 11 \\
\hline
\end{tabular}

on OPAL-RT Opal-RT eMEGAsim RTS. For the sake of reproducibility, the model is open-source and online available at [30]. It is worth noticing that the initialization procedure is important to ensure correct steady-state solution. In this regard, the detailed load flow based initialization procedure is given in [30].

a) Synchronous Generators: The conventional generation asset consists of both hydro- and thermal-power plants. For this analysis, we simulate the generators by means of a dynamic model of the prime mover, a synchronous machine, a speed governor, a DC1A excitation system [46] and an Automatic Voltage Regulator (AVR). For the synchronous machine, we adopt the sixth-order state-space model [47].

The generator model includes a Primary Frequency Regulator (PFR) with a droop coefficient of 5\%. In the thermal-power plant (G1), that accounts for the highest installed capacity, we implement also a Secondary Frequency Regulator (SFR), whose integration time constant is set equal to $120 \mathrm{~s}$.

As a summary, Table II reports the plant type, nominal capacity and frequency regulation for each generator.

b) Wind Generation: In Table II we show also the implementation details of the 4 wind farms. In this regard, the generator is modelled as Type-3 Doubly-Fed Induction Generator (DFIG) that consists of an asynchronous machine and a back-to-back Voltage Source Converter (VSC). In particular, the VSC behavior is reproduced by two equivalent voltage sources, which generate the $\mathrm{AC}$ voltage averaged over one cycle of the switching frequency. In this way, it is reasonable to expect that the dynamic interaction between renewable generation and UFLS control scheme is preserved [48].

For this analysis, we do not develop a detailed aerodynamic model of the wind turbine, as its effect is accounted already in the wind profiles. Indeed, the wind profiles are generated at 1 second resolution by re-sampling the measurements at 1 minute resolution from ERCOT [49]. The re-sampling approach is based on iterated smoothing and differentiating operations that use the statistical characteristics of the aggregated wind generation profiles presented in [50].

c) Dynamic Load Model: Static load models, including constant impedance, constant current model, and constant power models, are well known in the literature and can be easily implemented [51]. Nevertheless, such static models do not provide an accurate approximation of the load frequency and voltage responses. In order to reproduce a plausible dynamic load behavior, the EPRI LOADSYN model has been

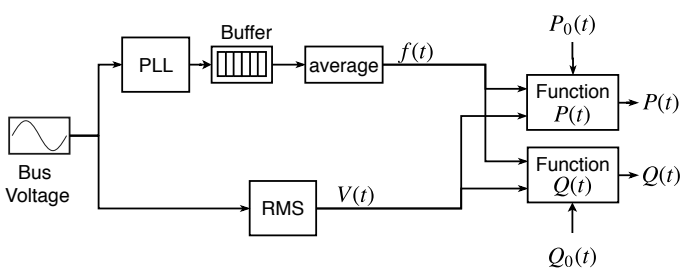

Fig. 3. Diagram of the EPRI LOADSYN dynamic load model.

adopted [52]. ${ }^{1}$ Specifically, we implemented the three-phase dynamic load model based on the following equations:

$$
\begin{aligned}
& P(t)=P_{0}(t)\left(\frac{V(t)}{V_{0}}\right)^{K_{p v}}\left[1+K_{p f}\left(f(t)-f_{0}\right)\right] \\
& Q(t)=Q_{0}(t)\left(\frac{V(t)}{V_{0}}\right)^{K_{q v}}\left[1+K_{q f}\left(f(t)-f_{0}\right)\right]
\end{aligned}
$$

where $P(t)$ and $Q(t)$ are the three-phase load active and reactive power. The parameters $K_{p v}=1.7, K_{p f}=1.0$, $K_{q v}=2.6, K_{q f}=-1.7$ are obtained from typical load voltage and frequency parameters inferred from EPRI LOADSYN program. In this regard, we represent $f(t), V(t), P_{0}(t)$, and $Q_{0}(t)$ as time-varying variables sampled with a resolution of $20 \mathrm{~ms}$. We assume that $P_{0}(t)$ and $Q_{0}(t)$ are active and reactive power consumed at rated frequency and voltage. The rated demand profile is adapted from a monitoring system based on PMUs installed on the $125 \mathrm{kV}$ sub-transmission system of Lausanne, Switzerland [53]. Coherently with the other model variables, the measured time-series power data are sampled with a resolution of $20 \mathrm{~ms}$. Since the nominal load values in the original IEEE 39-Bus power system are different from our measured data, the final demand patterns are obtained by re-scaling the measured time series.

The implementation of the EPRI LOADSYN model is illustrated in Fig. 3. A conventional Phase Locked Loop (PLL) and a Root Mean Square (RMS) operator measure the bus frequency and voltage to be employed in the dynamic load model. On one side, as the PLL may be inaccurate in transient conditions, a moving average mechanism is implemented in order to avoid improper behavior of the dynamic load model. Specifically, the PLL-tracked frequency is updated every $1 \mathrm{~ms}$, and then buffered for averaging. The overall buffer size is 240 samples, with an overlap size of 220 samples (i.e., the final frequency $f(t)$ is reported every $20 \mathrm{~ms}$ ). On the other side, the bus voltage $V(t)$ is given by a RMS operator reporting every $20 \mathrm{~ms}$. The RMS value is computed over a window length of $240 \mathrm{~ms}$, as to be consistent with the frequency estimation.

d) Phasor Measurement Units: In this paper, we compare the accuracy provided by two different ROCOF estimation techniques, based on consolidated state-of-the-art algorithms like e-IpDFT [27] and cs-TFM [28]. The details regarding their implementation within the adopted RTS are provided in [44] and [45], respectively.

The e-IpDFT PMU adopts an enhanced version of the IpDFT to estimate the synchrophasor associated to the fun-

\footnotetext{
${ }^{1}$ Although this paper adopts only the dynamic load model, a combination of dynamic and static load models may be also considered.
} 
damental component of the power signal under analysis. Such technique, described in Algorithm 2, is specifically designed to mitigate the effects of long-range spectral leakage produced by the negative image of the fundamental component.

\begin{tabular}{ll}
\hline Algorithm 2 e-IpDFT \\
\hline 1: & $x[n]:=\left\{x\left(t_{n}\right) \quad t_{n}=n T_{s}, n=[0, \ldots N-1] \in \mathbb{N}\right\}$ \\
2: & $X(k)=\operatorname{DFT}(x[n] \cdot w[n])$ \\
3: $\left\{\widehat{f}, \widehat{A}, \widehat{\varphi}_{0}\right\}=\operatorname{IpDFT}(X(k))$ \\
4: $\widehat{X}^{-}(k)=\mathrm{wf}\left(-\widehat{f}, \widehat{A},-\widehat{\varphi}_{0}\right)$ \\
5: $\widehat{X}^{+}(k)=X(k)-\widehat{X}^{-}(k)$ \\
6: $\left\{\widehat{f}, \widehat{A}, \widehat{\varphi}_{0}\right\}=\operatorname{IpDFT}\left(\widehat{X}^{+}(k)\right)$ \\
7: $\hat{R}=\operatorname{diff}(\hat{f}) / T_{r}$ \\
\hline
\end{tabular}

First, the PMU acquires a discrete time-series of samples $x[n]$, where $x(t)$ is the time-variant power system signal under analysis, $N$ is the number of samples contained in the considered observation interval and $F_{s}=T_{s}^{-1}$ is the sampling rate (line 1). The signal is windowed with the Hanning function $w[n]$ to reduce the long-range spectral leakage effects, then the weighted signal DFT $X(k)$ is computed (line 2).

The IpDFT technique applied to the highest DFT bins, provides a preliminary estimate of the fundamental parameters (line 3). With respect to the location of the highest amplitude bin $k_{m}$, the fractional correction term $\delta$ is given by:

$$
\delta=\varepsilon \cdot \frac{2 \cdot\left|X\left(k_{m}+\varepsilon\right)\right|-\left|X\left(k_{m}\right)\right|}{\left|X\left(k_{m}+\varepsilon\right)\right|+\left|X\left(k_{m}\right)\right|}
$$

and is used to refine the fundamental parameter estimates as:

$$
\begin{aligned}
\hat{A}=\left|X\left(k_{m}\right)\right|\left|\frac{\pi \delta}{\sin (\pi \delta)}\right|\left|\delta^{2}-1\right| & \hat{\varphi}_{0}=\angle X\left(k_{m}\right)-\pi \delta \\
\hat{f}=\left(k_{m}+\delta\right) F_{s} / N & \hat{R}=\operatorname{diff}(\hat{f}) / T_{r}
\end{aligned}
$$

These values enable us to reconstruct the component's negative image $\widehat{X}^{-}(k)$ (line 4), and subtract it from the original DFT bins, that now account only for the fundamental component's positive image $\widehat{X}^{+}(k)$ (line 5). In this reducedleakage scenario, we apply again the IpDFT for a further enhanced estimation of the fundamental parameters $\left\{\hat{f}, \hat{A}, \hat{\varphi}_{0}\right\}$ (line 6). Finally, we compute the fundamental ROCOF $\hat{R}$ as the finite difference between two consecutive frequency estimations, divided by the reporting period $T_{r}$ (line 7).

The cs-TFM PMU adopts a formulation of the TaylorFourier Transform (TFT), that has been suitably modified and generalized in order to deal also with multi-tone power signals. Thanks to a Taylor series expansion truncated to the second derivative order, it is possible to include the fundamental frequency and ROCOF within the estimator state variables as the first and second time-derivative of the phase angle, respectively. However, the TFT performance strongly depends on the spectral support $\mathcal{S}$ employed for the filter positioning: if any significant spectral component is neglected or badly identified, the TFT results might suffer from uncompensated spectral leakage and thus lead to inaccurate estimates [38].

The cs-TFM method recovers the spectral support $\mathcal{S}$ through an Orthogonal Matching Pursuit (OMP) algorithm, i.e. a greedy selection routine that exploits the assumption that the signal spectrum is sparse and consists only of a limited number of narrow-band components. The support recovery stage might suffer from the poor frequency resolution provided by DFT when short observation intervals are taken into account. In order to partially overcome this limitation, we apply a CSbased super-resolution technique that enables us to reduce the bin spacing by one order of magnitude.

Given the recovered support $\mathcal{S}$, we design the corresponding TFM model $M$ and we compute the dynamic synchrophasor $p$ that consists of three Taylor-Fourier series coefficients:

$$
p=\left\{p^{0}, p^{1}, p^{2}\right\}=\operatorname{pinv}(M) \cdot x
$$

where the superscript denotes the derivative order. Based on this, we are able to estimate the fundamental parameters as:

$$
\begin{aligned}
\hat{A}=\left|p^{0}\right|, & \hat{A}^{1}=2 \Re\left(p^{1} \cdot e^{-j \hat{\varphi}}\right) \\
\hat{\varphi}=\angle p^{0}, & \varphi^{1}=\frac{\Im\left(p^{1} \cdot e^{-j \hat{\varphi}}\right)}{\hat{A}} \\
\hat{f}=\hat{f}_{0}+\frac{\hat{\varphi}^{1}}{2 \pi}, & \hat{R}=\frac{\Im\left(p^{2} \cdot e^{-j \hat{\varphi}}\right)-\hat{A}^{1} \cdot \hat{\varphi}^{1}}{2 \pi \cdot \hat{A}}
\end{aligned}
$$

where $\hat{R}$ denotes the estimated ROCOF, and $f_{0}$ is the fundamental frequency within the recovered spectral support. The spectral analysis can be limited to the expected variation range of the fundamental component, i.e. $[45,55] \mathrm{Hz}$.

As shown in Algorithm 3, the first step consists in enhancing the frequency resolution by projecting $X$ over the vector space spanned by matrix $D_{f}$. In more detail, the matrix columns are designed to account for leakage effects over a super-resolved grid, whose bin spacing is set to $1.515 \mathrm{~Hz}$ (line 1).

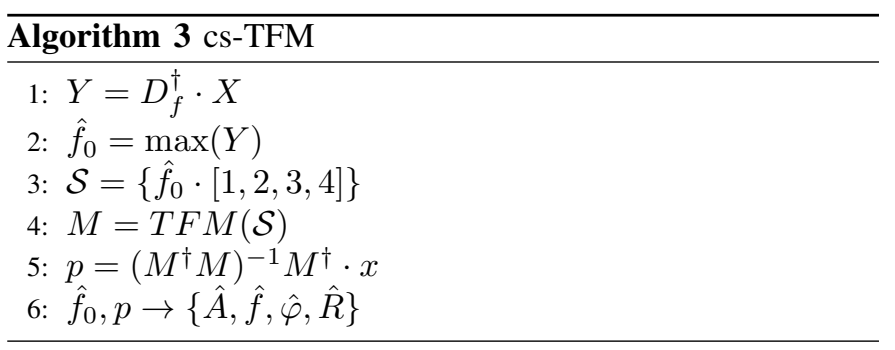

We associate the fundamental frequency $\hat{f}_{0}$ to the maximum bin of the super-resolved spectrum (line 2), and we include into the spectral support $\mathcal{S}$ the first four harmonic terms (line 3). Based on this information, we construct the TFM matrix (line 4) and compute the corresponding dynamic phasor coefficients (line 5). Finally, by applying (7), we extract the fundamental synchrophasor, frequency and ROCOF (line 6).

e) On the Tuning of the UFLS Scheme Parameters: In order to demonstrate how post-contingency voltage modulations interfere with the ROCOF estimation, we present in Fig. 4 the voltage waveform and the corresponding ROCOF measurements in the IEEE 39-bus power grid experiencing a large contingency. Specifically, at $t=180 \mathrm{~s}$ a total amount of $1.5 \mathrm{GW}$ generation power is tripped. The waveforms refer to bus 26, but similar considerations hold for the rest of the buses. The ROCOF estimates are provided by 4 PMUs: for both e-IpDFT and cs-TFM, we implement two different configurations, as representative of P- and M-class of IEEE Std c37.118.1 [7]. By means of the curve fitting tool provided 


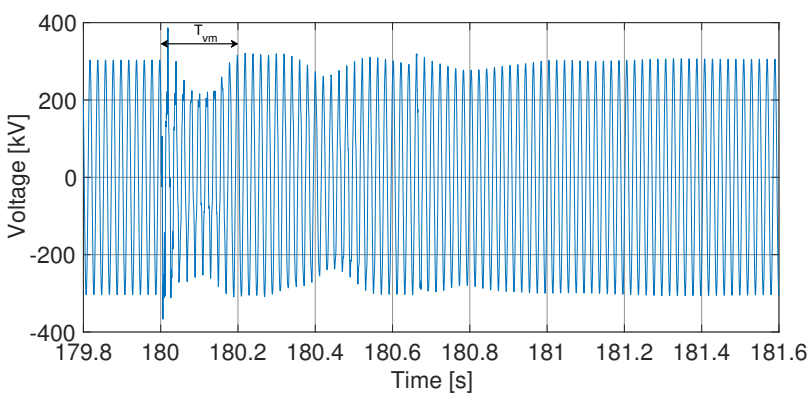

(a)

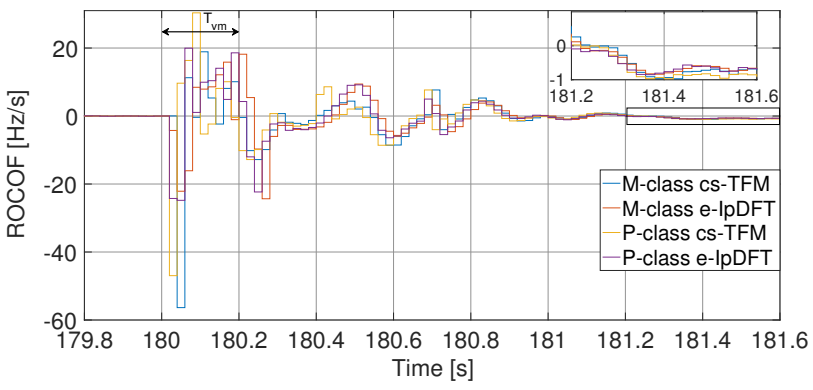

(b)

Fig. 4. Example of voltage modulation interfering with ROCOF estimation. Voltage waveform (a); ROCOF measurements (b).

by MATLAB, we are able to fit the waveform with a model consisting of a sum of sines (one representing the fundamental tone, two for the amplitude modulation, two for the phase modulation). Thereby we characterize the modulations of the waveform in terms of depth and frequency: $12.90 \%$ and 5.27 $\mathrm{Hz}$ for the amplitude modulation, and $153 \mathrm{mrad}$ and 4.23 $\mathrm{Hz}$ for the phase modulation. In the first modulation period $T_{V M}$, the voltage modulation significantly affects the ROCOF estimation, and the 4 PMUs provide unreliable results (refer to Fig. 4b). Conversely, the ROCOF measurements become way more consistent when the voltage modulation is damped, as illustrated in the zoomed window in Fig. 4b. Therefore, it is recommended to wait for a proper time interval before relying on a ROCOF estimate. In the present paper, we adopt a 500 ms observation interval, as recommended in [42].

A similar sensitivity study enables us to tune the ROCOF $R$ and the activation thresholds $p_{a c t}$, as a function of the severity of the power outage. Specifically, we conduct dedicated simulations for the tripping of $1.0 \mathrm{GW}, 1.25 \mathrm{GW}, 1.5 \mathrm{GW}$, $1.75 \mathrm{GW}$ and we analyze the frequency dynamics after these critical events. Fig. 5 shows the simulation results, as reported by a P-class e-IpDFT PMU at bus 26 (similar results hold for all buses and for all PMUs). As illustrated in Fig. 5b, the most severe contingency corresponds to the fastest frequency decrease, i.e., larger ROCOF. Briefly, the larger the measured ROCOF, the larger the detected contingency and, therefore, the larger the amount of loads to be shed. This is consistent with the ROCOF thresholds $R$ in Table I. In a similar way, also the activation thresholds $p_{\text {act }}$ are tuned with respect to the contingency severity. As reported in Table I, the larger the detected contingency, the faster the UFLS should act, therefore, the lower the activation threshold.

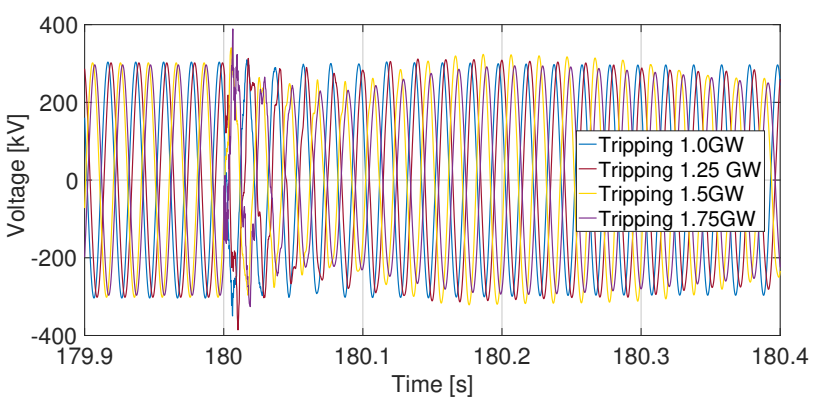

(a)

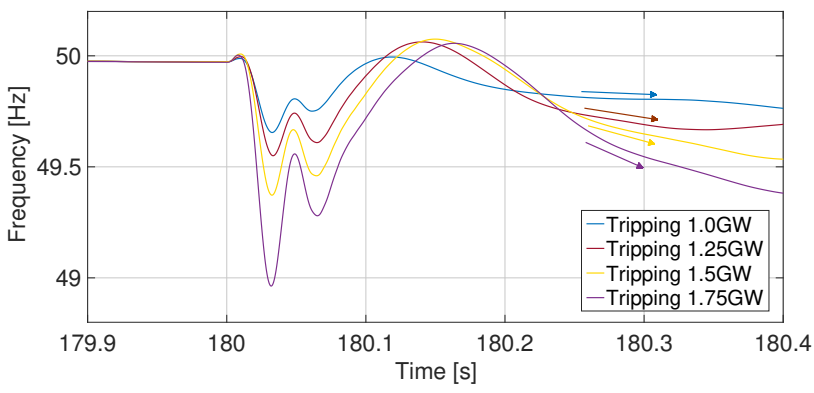

(b)

Fig. 5. Sensitivity study results. Voltage waveform on bus 26 (a); Frequency measurements on bus 26 by P-class e-IpDFT PMU (b).

\section{VAlidation RESUlts}

In this section, we numerically assess the performance of the proposed UFLS policy, with a focus on the impact of the synchrophasor estimation algorithm and of the UFLS parameters. As described in Section IV, the strategy is embedded within the modified IEEE 39-bus. Specifically, we simulate a large contingency event, in which G4, G6 and G7 (1.5 GW total generation) are tripped at $t=180 \mathrm{~s}$. For each synchrophasor estimation algorithm, we implement two different configurations, as representative of $\mathrm{M}$ - and P-class of IEEE Std c37.118.1 [7]. In order to study whether different thresholds can affect the performance of the overall control scheme, the tests are repeated with both $R$-LS- 1 and $R$-LS-2. The simulation results for $R$-LS- 1 an $R$-LS-2 are respectively shown in Fig. 6 and Fig. 7, in terms of measured ROCOF and active power profile. For the sake of clarity, in the following graphs we consider a single representative load for each class, i.e., load 16 for M-class and load 4 for P-class.

Let us first consider the ROCOF measurements from the 4 PMUs. For M-class configuration, Fig. 6a and 7a show the ROCOF measurements provided by cs-TFM and e-IpDFT for $R$-LS-1 and $R$-LS-2, respectively. As for P-class configuration, Fig. $6 \mathrm{c}$ and Fig. 7c show the ROCOF measurements provided by cs-TFM and e-IpDFT for $R$-LS- 1 and $R$-LS-2, respectively. As shown in the figures, right after the contingency, the csTFM estimates of ROCOF anticipate the e-IpDFT ones by one reporting period (i.e. $20 \mathrm{~ms}$ ). This anticipatory effect is due to the fact that cs-TFM adopts a dynamic signal model that allows for a direct estimation of the phase second-order timederivative, whereas e-IpDFT adopts a static signal model and calculates ROCOF as the finite-difference between subsequent 


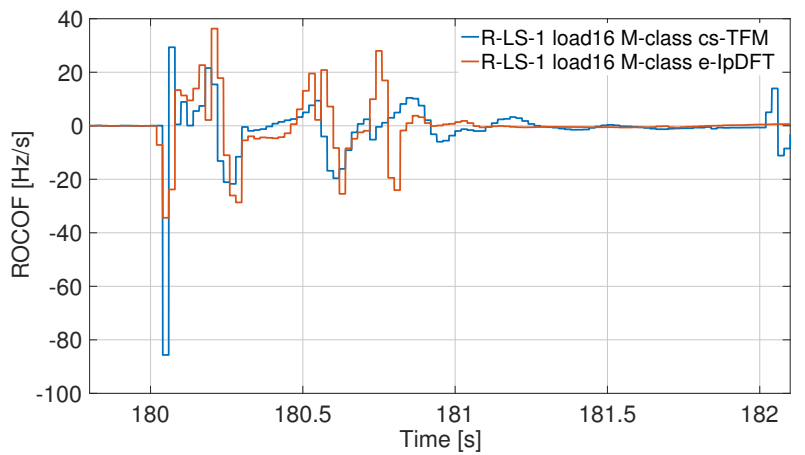

(a)

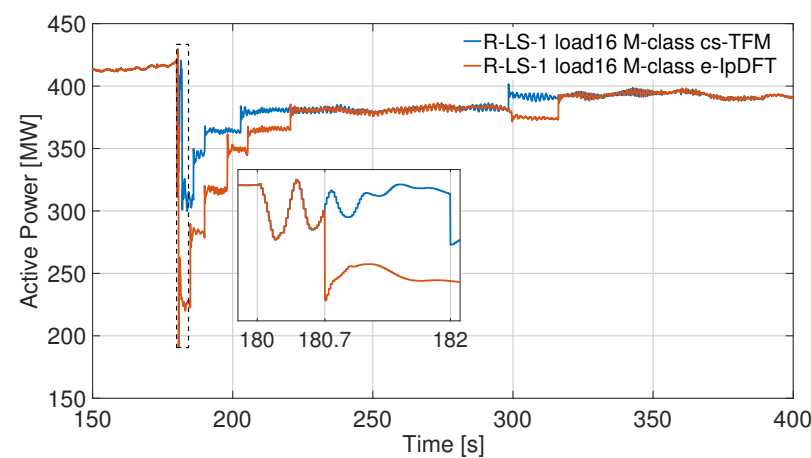

(b)

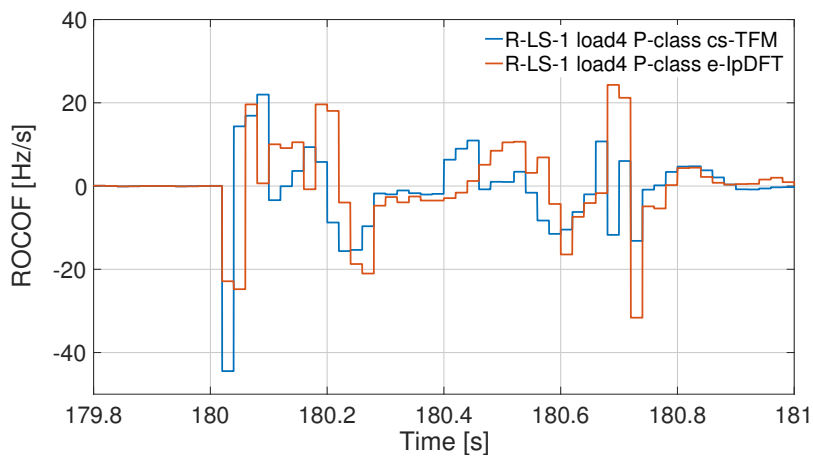

(c)

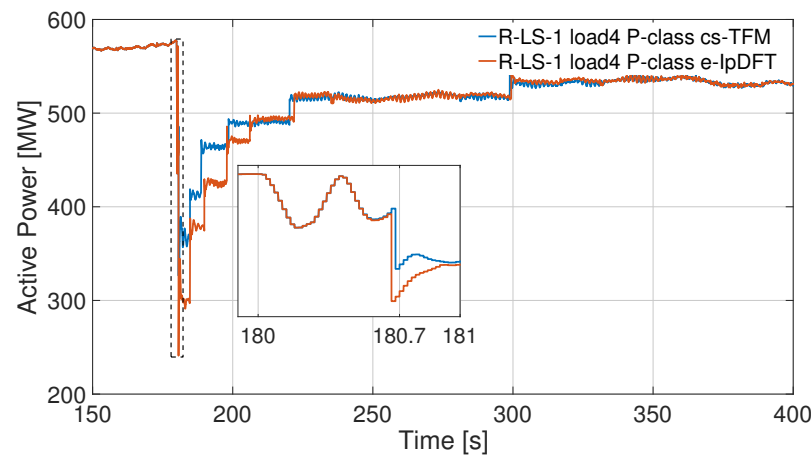

(d)

Fig. 6. PMU-based ROCOF measurements and corresponding active power profiles for $R$-LS-1 thresholds: (a) and (b) refer to load 16 and M-class configuration, whereas (c) and (d) refer to load 4 and P-class configuration.

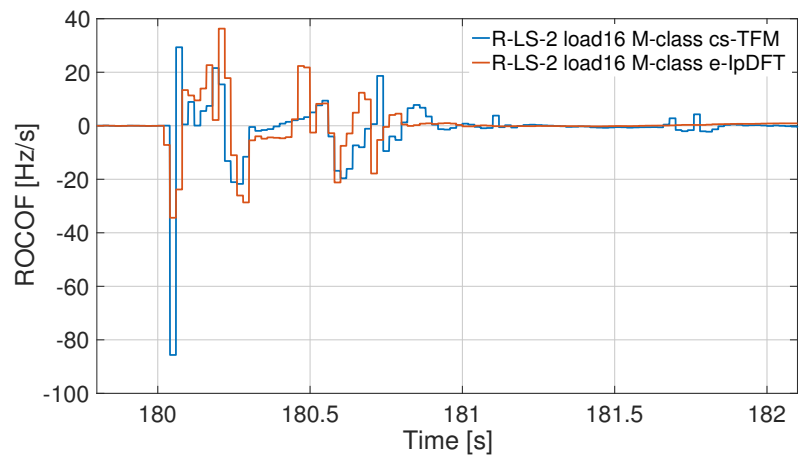

(a)

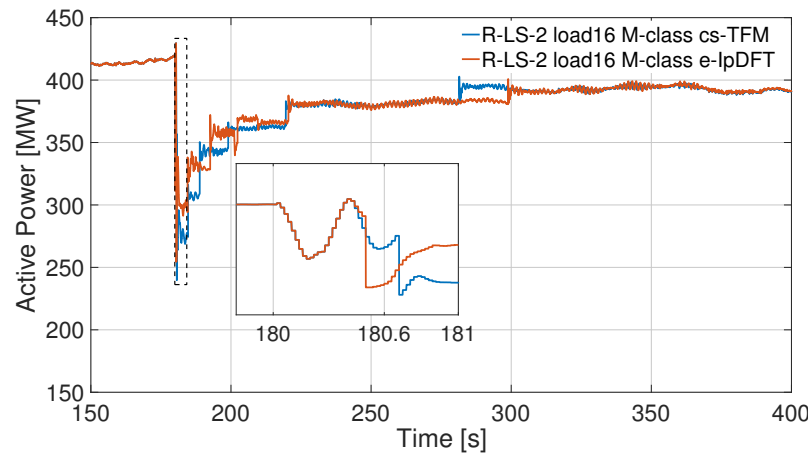

(b)

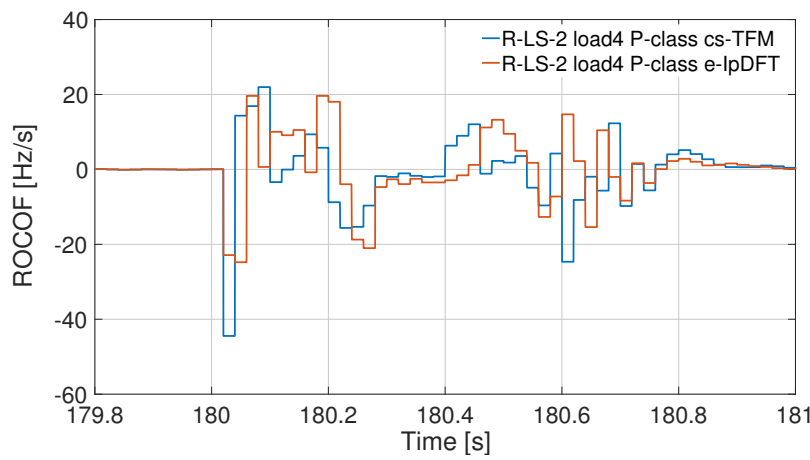

(c)

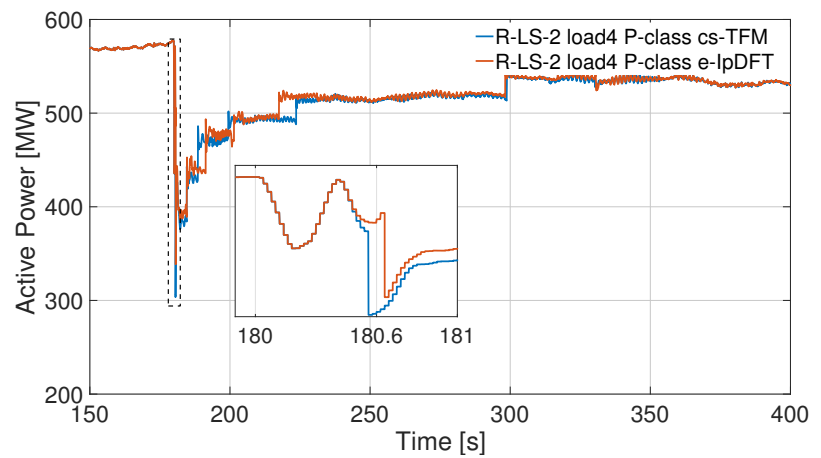

(d)

Fig. 7. PMU-based ROCOF measurements and corresponding active power profiles for $R$-LS-2 thresholds: (a) and (b) refer to load 16 and M-class configuration, whereas (c) and (d) refer to load 4 and P-class configuration. 
frequency measurements This is particularly noticeable within the first $300 \mathrm{~ms}$ after the contingency. As ROCOF-LS starts to be triggered, this effect is less visible.

The corresponding active powers are displayed in Fig. 6b, Fig. 7b, Fig. 6d and Fig. 7d, and illustrate the control actions of $R$-LS and $f$-LR control scheme. Given $R$-LS-1 thresholds for M-class configuration, the zoomed window in Fig. 6b shows that the e-IpDFT sheds a larger share of loads $(+25 \%) 1.3 \mathrm{~s}$ earlier than the cs-TFM. Given $R$-LS-2 thresholds for M-class configuration, the zoomed window in Fig. $7 \mathrm{~b}$ shows that the same amount of loads is shed by both cs-TFM and e-IpDFT, yet the e-IpDFT sheds earlier and the cs-TFM determines a faster system restoration. Briefly, using both $R$-LS-1 and $R$ LS-2, e-IpDFT sheds earlier whereas cs-TFM restores before. Although the cs-TFM estimates of ROCOF are anticipated by one reporting period, the e-IpDFT ones are characterized by higher ROCOF values. Therefore, in accordance with the threshold in Table I, it is reasonable to expect that the e-IpDFT estimates produce a larger amount of loads to be shed (refer to Fig. 6a and Fig. 7a). As for $R$-LS-2 thresholds, this difference is less significant since the more conservative thresholds in Table I lead to faster load shedding for both estimators.

Given the $R$-LS-1 and $R$-LS-2 thresholds for P-class configuration, Fig. $6 \mathrm{~d}$ shows that the e-IpDFT sheds a larger share of loads $(+10 \%)$ and Fig. $7 \mathrm{~d}$ shows that the same amount of loads is shed by both cs-TFM and e-IpDFT. As shown in the zoomed windows, for both the estimators the load shedding is triggered earlier in P-class than in M-class configuration. This is due to the fact that, given the shorter observation interval of P-class PMUs, both estimators provide less accurate results. Specifically, their ROCOF estimates are quite similar and, therefore, leading to a similar amount of LS (refer to Fig. 6c and Fig. 7c).

In view of a quantitative evaluation of the performance of the considered ROCOF estimators, in each simulated scenario we have computed the Expected Energy Not Served (EENS) ${ }^{2}$ and the Integrated Frequency Variation (IFV) ${ }^{3}$. In this context, Table III reports their values as accumulated over all the load buses of the simulated power system.

The thresholds' comparison shows that $R$-LS-2 corresponds to higher EENS values than $R$-LS-1. Indeed, given the same ROCOF thresholds, the amount of loads to be shed is higher in $R$-LS-2 scenario. As discussed in III, the $R$-LS- 2 thresholds are associated with lower ROCOF values and activation limits. Coherently, this implementation choice produces much higher EENS values. As regards IFV, we would expect that a higher LS share corresponds to a lower IFV, thanks to the reduction of the frequency drop. However, if an excessive amount of loads is shed, it is possible to experience a subsequent rapid frequency increase, not properly compensated by LR actions. An example of this phenomenon is shown in Fig. 8, where we compare the evolution of frequency measurements as provided by all four PMU configurations in $R$-LS-1 and $R$-LS-2. The

\footnotetext{
${ }^{2}$ EENS refers to the expected amount of energy not being served to the demand during the UFLS and LR actions.

${ }^{3}$ IFV refers to the total integrated frequency deviation (absolute value) of all the load buses during the UFLS and LR actions.
}

TABLE III

UFLS SCheme PeRformance In Simulated SCenarios

\begin{tabular}{ccccc}
\hline Threshold & Estimator & Class & EENS [MWh] & IFV \\
\hline \multirow{4}{*}{$R$-LS-1 } & cs-TFM & M & 14.05 & 1336.2 \\
& e-IpDFT & M & 16.70 & 1358.4 \\
& cs-TFM & P & 14.51 & 1329.3 \\
& e-IpDFT & P & 16.83 & 1373.1 \\
\hline \multirow{4}{*}{$R$-LS-2 } & cs-TFM & M & 14.21 & 1334.4 \\
& e-IpDFT & M & 24.17 & 1568.8 \\
& cs-TFM & P & 19.23 & 1383.2 \\
& e-IpDFT & P & 24.20 & 1458.9 \\
\hline
\end{tabular}

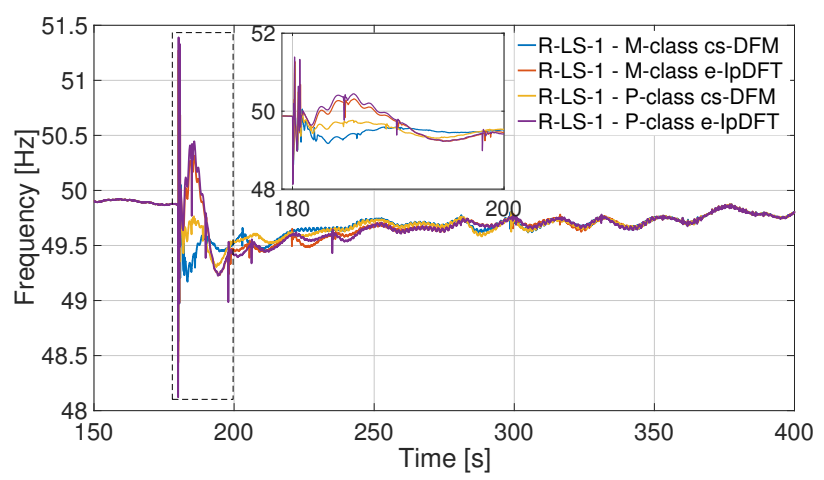

(a)

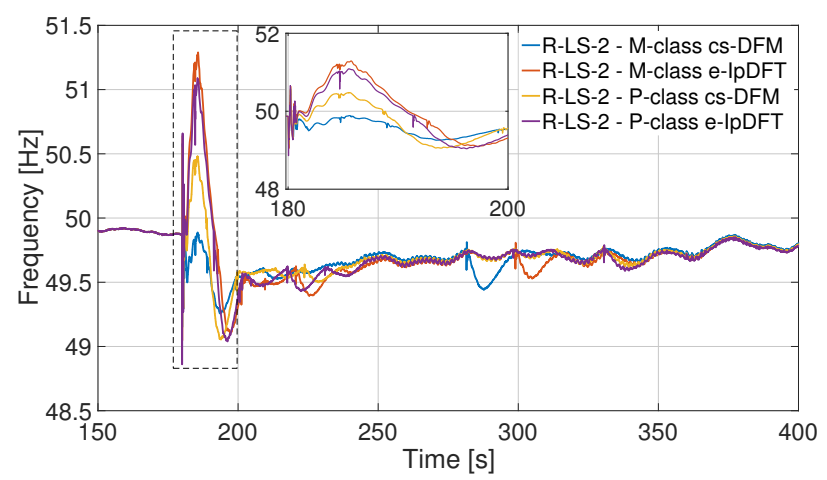

(b)

Fig. 8. Frequency: M-class cs-TFM (blue), M-class e-IpDFT (orange), P-class cs-TFM (yellow), P-class e-IpDFT (violet). Frequency for thresholds $R$-LS-1 (a); Frequency for thresholds $R$-LS-2 (b).

frequency overshoots are clearly visible in the zoomed window in Fig. 8b.

In general, as shown in Fig. 6 and Fig. 7, the cs-TFM algorithm allows for an instantaneous estimation of ROCOF, thanks to the direct computation of the phase second-order time-derivative. The anticipation of one reporting period (i.e. $20 \mathrm{~ms}$ ) guarantees a more consistent response to fast dynamics. Based on the results of Table III, it is possible to infer some recommendations for the implementation of the proposed UFLS schemes: it is important also to define proper $R$-LS thresholds in order to minimize EENS and IFV, as well as to avoid excessive LS and frequency overshoots.

\section{CONCLUSIONS}

In this paper, we investigated the impact of synchrophasor estimation algorithms in ROCOF-based UFLS schemes. To 
this end, we developed a simple yet effective local UFLS and Load-Restoration (LR) scheme, that relies on PMU-based estimates of fundamental frequency and ROCOF. Specifically, we introduce two sets of ROCOF thresholds to promptly trig the LS and suitably select the amount of shed loads. Frequency measurements, instead, govern the LR process. The employment of PMUs enables us to optimize the estimation accuracy and the reporting rate. In addition, differently from traditional approaches based on frequency thresholds, the proposed scheme employs ROCOF measurements to trig the LS action, as its derivative formulation allows for a prompter and more effective response to the fast dynamics experienced in modern power systems with reduced inertia.

We assessed the performance of the proposed relaying scheme by means of a RTS, where we reproduced a fullreplica of the time-domain dynamic model of the IEEE 39-Bus power system with a substantial amount of distributed energy resources. In this context, we considered two consolidated synchrophasor estimation algorithms, i.e., e-IpDFT and cs-TFM, that rely on a static and a dynamic signal model, respectively. For each algorithm, we implemented two configurations as representative of P- and M-class of IEEE Std C37.118.1.

Within this simulated scenario, we reproduced a contingency event with $1.5 \mathrm{GW}$ tripped power, and we evaluated the UFLS and LR performance for each combination of RO$\mathrm{COF}$ thresholds and algorithm configurations. As performance metrics, we considered both the Expected Energy Not Served (EENS) and the Integrated Frequency Variation (IFV).

In M-class configuration, the ROCOF variation range is limited and the dynamic model approach provides a prompter system restoration. In P-class configuration, instead, we notice higher ROCOF estimations leading to higher EENS and IFV.

The comparison between different ROCOF thresholds shows that high shares of shed loads might lead to an uncontrolled frequency increase and thus trig a too fast and excessive LR action.

In conclusion, the proposed analysis confirmed the potential benefits of PMU-based ROCOF measurements for UFLS applications, and proved that the control scheme performance depends on the adopted synchrophasor estimation technique and configuration.

\section{REFERENCES}

[1] "IEEE guide for the application of protective relays used for abnormal frequency load shedding and restoration," IEEE Std C37.117-2007, pp. 1-55, Aug 2007.

[2] ENTSO-E, "Technical background and recommendations for defence plans in the continental Europe synchronous area," in Tech. Rep., 2010.

[3] B. Delfino, S. Massucco, A. Morini, P. Scalera, and F. Silvestro, "Implementation and comparison of different under frequency loadshedding schemes," in 2001 PES Summ Meet, vol. 1, July 2001, pp. 307-312 vol.1.

[4] AEMO, "Review of the black system south australia report system event of 28 september 2016," Australian Energy Market Operator, Tech. Rep., 2017.

[5] M. Karimi, P. Wall, H. Mokhlis, and V. Terzija, "A new centralized adaptive underfrequency load shedding controller for microgrids based on a distribution state estimator," IEEE Trans on Pow Del, vol. 32, no. 1, pp. 370-380, Feb 2017

[6] U. Rudez and R. Mihalic, "Wams-based underfrequency load shedding with short-term frequency prediction," IEEE Trans on Pow Del, vol. 31, no. 4, pp. 1912-1920, Aug 2016.
[7] "IEEE Standard for Synchrophasor Measurements for Power Systems," IEEE Std C37.118.1-2011 (Revision of IEEE Std C37.118-2005), pp. 1-61, Dec 2011.

[8] "IEEE Standard for Synchrophasor Measurements for Power Systems - Amendment 1: Modification of Selected Performance Requirements," IEEE Std C37.118.1a-2014 (Amendment to IEEE Std C37.118.1-2011), pp. 1-25, April 2014

[9] A. G. Phadke and B. Kasztenny, "Synchronized phasor and frequency measurement under transient conditions," IEEE Trans on Pow Del, vol. 24, no. 1, pp. 89-95, Jan 2009.

[10] G. Frigo, D. Colangelo, A. Derviškadić, M. Pignati, C. Narduzzi, and M. Paolone, "Definition of accurate reference synchrophasors for static and dynamic characterization of pmus," IEEE Trans on Instrum and Meas, vol. 66, no. 9, pp. 2233-2246, Sep. 2017.

[11] A. J. Roscoe and al., "The case for redefinition of frequency and ROCOF to account for AC power system phase steps," in 2017 IEEE AMPS, Sept 2017, pp. 1-6.

[12] G. Frigo, A. Derviškadić, Y. Zuo, and M. Paolone, "PMU-based rocof measurements: Uncertainty limits and metrological significance in power system applications," IEEE Transactions on Instrumentation and Measurement, pp. 1-1, 2019.

[13] R. M. Gardner and Y. Liu, "Generation-load mismatch detection and analysis," IEEE Transactions on Smart Grid, vol. 3, no. 1, pp. 105-112, March 2012.

[14] Kulkarni, J. Payne, and P. Mistretta, "Integrating scada, load shedding, and high-speed controls on an ethernet network at a north american refinery," IEEE Transactions on Industry Applications, vol. 51, no. 2, pp. 1360-1368, March 2015.

[15] A. Raj, G. Gajjar, and S. A. Soman, "Controlled islanding of transmission system using synchrophasor measurements," IET Generation, Transmission Distribution, vol. 13, no. 10, pp. 1942-1951, 2019.

[16] A. Chakrabortty, J. H. Chow, and A. Salazar, "Interarea model estimation for radial power system transfer paths with intermediate voltage control using synchronized phasor measurements," IEEE Transactions on Power Systems, vol. 24, no. 3, pp. 1318-1326, Aug 2009.

[17] G. Xu and V. Vittal, "Slow coherency based cutset determination algorithm for large power systems," IEEE Transactions on Power Systems, vol. 25, no. 2, pp. 877-884, May 2010.

[18] H. Bevrani, G. Ledwich, and J. J. Ford, "On the use of df/dt in power system emergency control," in 2009 IEEE/PES Pow Sys Conf, March 2009, pp. 1-6.

[19] L. Sigrist, "A UFLS scheme for small isolated power systems using rate-of-change of frequency," IEEE Trans on Pow Sys, vol. 30, no. 4, pp. 2192-2193, July 2015.

[20] C. P. Reddy, S. Chakrabarti, and S. C. Srivastava, "A sensitivitybased method for under-frequency load-shedding," IEEE Transactions on Power Systems, vol. 29, no. 2, pp. 984-985, March 2014.

[21] P. M. Anderson and M. Mirheydar, "An adaptive method for setting underfrequency load shedding relays," IEEE Trans on Pow Sys, vol. 7, no. 2, pp. 647-655, May 1992.

[22] U. Rudez and R. Mihalic, "Analysis of underfrequency load shedding using a frequency gradient," IEEE Trans on Pow Del, vol. 26, no. 2, pp. 565-575, April 2011.

[23] V. Terzija, "Adaptive underfrequency load shedding based on the magnitude of the disturbance estimation," IEEE Trans on Pow Sys, vol. 21 , no. 3, pp. 1260-1266, Aug 2006.

[24] B. Hoseinzadeh, F. F. da Silva, and C. L. Bak, "Decentralized coordination of load shedding and plant protection considering high share of RESs," IEEE Trans on Pow Sys, vol. 31, no. 5, pp. 3607-3615, Sept 2016.

[25] A. Saffarian and M. Sanaye-Pasand, "Enhancement of power system stability using adaptive combinational load shedding methods," IEEE Trans on Pow Sys, vol. 26, no. 3, pp. 1010-1020, Aug 2011.

[26] J. Tang, J. Liu, F. Ponci, and A. Monti, "Adaptive load shedding based on combined frequency and voltage stability assessment using synchrophasor measurements," IEEE Trans on Pow Sys, vol. 28, no. 2, pp. 2035-2047, May 2013.

[27] P. Romano and M. Paolone, "Enhanced interpolated-dft for synchrophasor estimation in fpgas: Theory, implementation, and validation of a pmu prototype," IEEE Trans on Instr and Meas, vol. 63, no. 12, pp. 2824-2836, Dec 2014.

[28] M. Bertocco, G. Frigo, C. Narduzzi, C. Muscas, and P. A. Pegoraro, "Compressive sensing of a taylor-fourier multifrequency model for synchrophasor estimation," IEEE Trans on Instr and Meas, vol. 64, no. 12, pp. 3274-3283, Dec 2015.

[29] R. Byerly, D. Sherman, and R. Bennon, "Phase II: frequency domain analysis of low-frequency oscillations in large electric power systems. 
Volume 1. Basic concepts, mathematical models, and computing methods." Westinghouse Electric Corp., Pittsburgh, PA (USA). Advanced Systems, Tech. Rep., 1982.

[30] DESL-EPFL, “IEEE-39-bus-4WG-power-system," 2018, accessed: 2019-07-12. [Online]. Available: https://github.com/DESL-EPFL/IEEE39-bus-4WG-power-system

[31] W. Dickerson, "Effect of PMU analog input section performance on frequency and ROCOF estimation error," in 2015 IEEE AMPS, Sept 2015, pp. 31-36.

[32] A. Riepnieks and H. Kirkham, "Rate of change of frequency measurement," in 2016 57th International Scientific Conference on Power and Electrical Engineering of Riga Technical University (RTUCON), Oct 2016, pp. $1-5$.

[33] A. Derviškadić, G. Frigo, and M. Paolone, "Beyond Phasors: Continuous-Spectrum Modeling of Power Systems using the Hilbert Transform," arXiv preprint arXiv:1906.11154, 2019.

[34] I. Kamwa, S. R. Samantaray, and G. Joos, "Wide frequency range adaptive phasor and frequency PMU algorithms," IEEE Trans on Smart Grid, vol. 5, no. 2, pp. 569-579, March 2014.

[35] "Application of phasor measurement units for monitoring power system dynamic performance," Cigré Working Group C4.34, Tech. Rep., 2017.

[36] "Time Synchronization in the Electric Power System," NASPI Time Synchronization Task Force, 2017.

[37] W. Yao, Y. Liu, D. Zhou, Z. Pan, M. J. Till, J. Zhao, L. Zhu, L. Zhan, Q. Tang, and Y. Liu, "Impact of GPS signal loss and its mitigation in power system synchronized measurement devices," IEEE Transactions on Smart Grid, vol. 9, no. 2, pp. 1141-1149, March 2018.

[38] C. Narduzzi, M. Bertocco, G. Frigo, and G. Giorgi, "Fasttfmmultifrequency phasor measurement for distribution networks," IEEE Trans on Instr and Meas, vol. 67, no. 8, pp. 1825-1835, Aug 2018.

[39] F. Saccomanno, Electric Power Systems: Analysis and Control. WileyIEEE Press, 2003

[40] Y. Wang, I. R. Pordanjani, W. Li, W. Xu, and E. Vaahedi, "Strategy to minimise the load shedding amount for voltage collapse prevention," IET Generation, Transmission Distribution, vol. 5, no. 3, pp. 307-313, March 2011.

[41] A. Derviškadić, Y. Zuo, G. Frigo, and M. Paolone, "Under frequency load shedding based on PMU estimates of frequency and ROCOF," in 2018 IEEE ISGT Europe, Oct 2018, pp. 1-6.

[42] ENTSO-E, "Rate of change of frequency (ROCOF) withstand capability," in Tech. Rep., 2017.

[43] R. A. Ramos et al., "Benchmark systems for small-signal stability analysis and control," IEEE Power and Energy Society, Tech. Rep, 2015.

[44] P. Romano, M. Pignati, and M. Paolone, "Integration of an IEEE Std. C37.118 compliant PMU into a real-time simulator," in 2015 IEEE Eindhoven PowerTech, June 2015, pp. 1-6.

[45] G. Frigo, A. Derviškadić, Y. Zuo, and M. Paolone, "Taylor-fourier model on a real-time simulator: Design, implementation and characterization," proccedding of the 2019 IEEE Powertech, Milano. available online: https://infoscience.epfl.ch/record/265966?\&ln=en.

[46] "IEEE draft recommended practice for excitation system models for power system stability studies," IEEE P421.5/D38, October 2015, pp. 1-202, Jan 2015.

[47] "IEEE guide for synchronous generator modeling practices and applications in power system stability analyses," IEEE Std 1110-2002 (Revision of IEEE Std 1110-1991), pp. 1-72, 2003.

[48] R. Pena, J. C. Clare, and G. M. Asher, "Doubly fed induction generator using back-to-back PWM converters and its application to variable-speed wind-energy generation," IEEE Proceedings, vol. 143, no. 3, pp. 231241, May 1996

[49] GE Energy, "Analysis of wind generation impact on ercot ancillary services requirements," 2008.

[50] K. Coughlin and J. H. Eto, "Analysis of wind power and load data at multiple time scales," Lawrence Berkeley National Laboratory (LBNL), Berkeley, CA (United States), Tech. Rep., 2010.

[51] T. Sadamoto, A. Chakrabortty, T. Ishizaki, and J. Imura, "Dynamic modeling, stability, and control of power systems with distributed energy resources: Handling faults using two control methods in tandem," IEEE Control Systems Magazine, vol. 39, no. 2, pp. 34-65, April 2019.

[52] "Load representation for dynamic performance analysis (of power systems)," IEEE Trans on Pow Sys, vol. 8, no. 2, pp. 472-482, May 1993.

[53] A. Derviškadić, P. Romano, M. Pignati, and M. Paolone, "Architecture and experimental validation of a low-latency phasor data concentrator," IEEE Trans on Smart Grid, vol. 9, no. 4, pp. 2885-2893, July 2018.

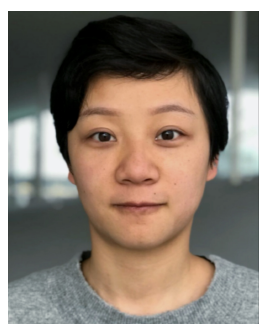

Yihui Zuo (S'18) was born in Sichuan, China, in 1992. She received the B.Sc. and M.Sc. degrees in electrical engineering from North China Electric Power University, Beijing, China, in 2013 and 2016, respectively. She is currently pursuing the Ph.D. degree with the Distributed Electrical System Laboratory, Swiss Federal Institute of Technology of Lausanne, Lausanne, Switzerland. Her current research interests include the impact of battery energy storage on the dynamics of power system with increasing resources interfaced with power electronics.

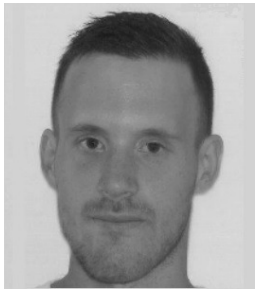

Guglielmo Frigo (M'18) was born in Padua, Italy, in 1986. He received the B.Sc. and M.Sc. degrees in biomedical engineering from the University of Padova, Padua, in 2008 and 2011, respectively, and the Ph.D. degree from the School of Information Engineering, University of Padova, in 2015, with a dissertation about compressive sensing (CS) theory applications to instrumentation and measurement scenario. He joined the Electronic Measurement Research Group, Department of Information Engineering, Padova, in 2011, and collaborated in the successful development of CS-based algorithms in the fields of spectral analysis, biomedical engineering, and smart-grid measurement. Since 2018 , he has been with the Distributed Electrical Laboratory, Swiss Federal Institute of Technology of Lausanne, Lausanne, Switzerland. He has co-authored several conference and journal papers on these topics. His current research interests include the development of enhanced measurement devices for active distribution networks.

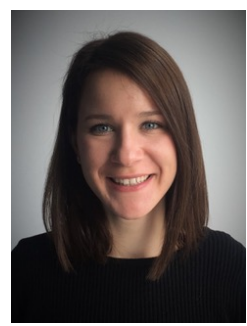

Asja Derviškadić (M'15) was born in Sarajevo, Bosnia and Herzegovina, in 1990. She received the B.Sc. and M.Sc. degrees (Hons.) in electrical engineering from the University of Rome "La Sapienza," Rome, Italy, in 2012 and 2015, respectively. She is currently pursuing the Ph.D. degree with the Distributed Electrical Systems Laboratory (DESL) of the Swiss Federal Institute of Technology of Lausanne (EPFL), Switzerland. Her research interests include the development of enhanced Phasor Measurements Units (PMUs) for active distribution

networks.

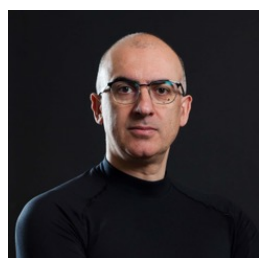

Mario Paolone (M'07, SM'10) received the M.Sc. (with Hons.) and Ph.D. degrees in electrical engineering from the University of Bologna, Italy, in 1998 and 2002, respectively. In 2005, he was appointed Assistant Professor in power systems with the University of Bologna, where he was with the power systems laboratory until 2011. In 2010, he received the Associate Professor eligibility from the Polytechnic of Milan, Italy. Since 2011, he joined the Swiss Federal Institute of Technology, Lausanne, Switzerland, where he is currently Full Professor, Chair of the Distributed Electrical Systems Laboratory, Head of the Swiss Competence Center for Energy Research Future Swiss Electrical infrastructure and Chair of the EPFL Energy Centre Directorate. He has authored or coauthored over 300 papers published in mainstream journals and international conferences in the area of energy and power systems. His research interests focus on power systems with particular reference to real-time monitoring and operation aspects, power system protections, dynamics and transients. Dr. Paolone is the Editor-in-Chief of the Elsevier journal Sustainable Energy, Grids and Networks. 